\title{
P05-I2. A computationally designed immunogen elicits potent anti-V3 neutralizing antibodies
}

\author{
CT Carrico*1, E Lagerquist ${ }^{2}$, E Boni ${ }^{3}$, YA Ban ${ }^{1}$, C Bretz ${ }^{3}$, K Ellingson ${ }^{2}$, \\ O Kalyuzhniy ${ }^{1}$, D Montefiori ${ }^{4}$, R Strong ${ }^{3}$, L Stamatatos ${ }^{2}$ and W Schief ${ }^{1}$
}

\author{
Address: ${ }^{1}$ Biochemistry, University of Washington, Seattle, WA, USA, ${ }^{2}$ Seattle Biomedical Research Institute, Seattle, WA, USA, ${ }^{3}$ Fred Hutchinson \\ Cancer Research Center, Seattle, WA, USA and ${ }^{4}$ Duke University, Durham, NC, USA \\ * Corresponding author
}

from AIDS Vaccine 2009

Paris, France. 19-22 October 2009

Published: 22 October 2009

Retrovirology 2009, 6(Suppl 3):P88 doi:10.1 186/1742-4690-6-S3-P88

This abstract is available from: http://www.retrovirology.com/content/6/S3/P88

(c) 2009 Carrico et al; licensee BioMed Central Ltd.

\section{Background}

Retrovaccinology aims to employ neutralizing monoclonal antibodies isolated from natural infection to guide design of immunogens that 're-elicit' similar neutralizing specificities. With that aim, we are developing computational immunogen design methods that take as input a crystal structure of an antibody-epitope complex and produce as output "epitope-scaffold" immunogens in which the antibody-bound structure of the epitope is transplanted onto and stabilized by scaffold proteins. We report our first results on scaffolding the V3 loop conformation bound by mAb 447D.

\section{Methods}

Computational methods were developed within the Rosetta modeling platform. Ten scaffolds were identified by structural search of the Protein Data Bank and subjected to backbone and sidechain grafting to transfer the 447D-bound epitope conformation.

\section{Results}

The three epitope-scaffolds with highest affinity for 447D had dissociation constants from 2 to $15 \mathrm{nM}$ as measured by surface plasmon resonance. These three exhibited specificity for 447D in that they showed little or no binding to 9 other anti-V3 monoclonals in Elisa. One epitope-scaffold, from human Interleukin-4, was selected for immunization. Rabbits (N:3) were immunized with epitopescaffold DNA ( 0 and 4 weeks; $0.5 \mathrm{mg}$ per animal per immunization) and then with epitope-scaffold protein $(12,19$ and 25 weeks; $0.05 \mathrm{mg}$ per animal per immunization) mixed with Ribi. Antisera collected after the proteinboost potently (IC50 > 250) neutralized HIV strains exposing the consensus clade B V3 sequence, but did not exhibit the full breadth of 447D itself.

\section{Conclusion}

The epitope-scaffold antisera represent the first potently neutralizing response to a computationally designed HIV antigen and demonstrate that computational design can achieve desired immunological effects. Iterative methods development with biophysical and immunological feedback promises to improve our ability to design immunogens that elicit predictable neutralizing responses.

\section{Acknowledgement}

This research was conducted as part of the Collaboration for AIDS Vaccine Discovery with support from the Bill \& Melinda Gates Foundation. 